\title{
$\beta$-Arrestin2 Inhibits the Apoptosis and Facilitates the Proliferation of Fibroblast-like Synoviocytes in Diffuse-type Tenosynovial Giant Cell Tumor
}

\author{
CHENXI CAO ${ }^{1,2,3 \#}$, YAN ZHANG ${ }^{4 \#}$, JIN CHENG $^{1,2,3}$, FEI WU ${ }^{1,2,3}$, XINGYUE NIU $^{1,2,3}$, XIAOQING HU $^{1,2,3}$, \\ XIAONING DUAN ${ }^{1,2,3}$, XIN FU $^{1,2,3}$, JIYING ZHANG $^{1,2,3}$, XIN ZHANG $^{1,2,3}$ and YINGFANG AO ZH, $^{1,2,3}$ \\ ${ }^{1}$ Department of Sports Medicine, Peking University Third Hospital, Beijing, P.R. China; \\ ${ }^{2}$ Institute of Sports Medicine of Peking University, Beijing, P.R. China; \\ ${ }^{3}$ Beijing Key Laboratory of Sports Injuries, Beijing, P.R. China; \\ ${ }^{4}$ Institute of Cardiovascular Sciences and Key Laboratory of Molecular Cardiovascular Sciences, \\ School of Basic Medical Sciences, Ministry of Education, Peking University Health Science Center, Beijing, P.R. China
}

\begin{abstract}
Background/Aim: Diffuse-type tenosynovial giant cell tumor (TGCT) is a rare benign proliferative synovial neoplasm of uncertain etiology, and the efficacy of surgical resection is not satisfactory. Therefore, there is an urgent need to explore the pathogenesis and identify novel therapeutic targets for TGCT. Materials and Methods: Synovial tissues were collected from patients with TGCT and osteoarthritis (OA). Differences of mRNA expression between TGCT and OA were explored using $m R N A$-seq. In addition, fibroblast-like synoviocytes (FLS) were treated with small interfering RNA (siRNA) or adenovirus in order to knockdown or overexpress $\beta$-arrestin2 (Arrb2), respectively. FLS proliferation and apoptosis were evaluated using the MTT assay and the caspase 3 activity assay, respectively. Results: The expression of Arrb2 in TGCT was significantly higher than that in OA. The overexpression of Arrb2 promoted the proliferation of FLS and inhibited its apoptosis, while knocking down Arrb2 had the opposite effect. Further
\end{abstract}

This article is freely accessible online.

\#These Authors equally contributed to this study.

Correspondence to: Yingfang Ao, Department of Sports Medicine, Peking University Third Hospital, 49 North Garden Road, Haidian District, Beijing 100191, P.R. China. Tel: +86 1082267390, Fax: +861062010440, e-mail: aoyingfang@163.com; Xin Zhang, Department of Sports Medicine, Peking University Third Hospital, 49 North Garden Road, Haidian District, Beijing 100191, P.R. China. Tel: +86 1082267390, Fax: +86 1062010440, e-mail: tozhangxin@sina.com

Key Words: Tenosynovial giant cell tumor, $\beta$-arrestin2, proliferation, apoptosis, targeted therapy. studies showed that Arrb2 can activate the PI3K-Akt signaling pathway, leading to increased proliferation of TGCT. Conclusion: Arrb2 facilitates the proliferation and inhibits the apoptosis of TGCT FLS through activating the PI3K-Akt cell survival pathway, providing new insight into the molecular mechanism of TGCT.

Diffuse-type tenosynovial giant cell tumor (TGCT) is a rare benign proliferative synovial neoplasm characterized by villous and nodular lesions of an uncertain etiology (1). The main clinical features of TGCT are synovial hyperplasia and recurrence. The hyperplastic synovial tissue will produce a large amount of bloody synovial fluid and cause excessive swelling of the joints, further causing joint pain and dysfunction (2). Uncontrollable excessive synovial proliferation can even cause malignant transformation of TGCT and result in amputation (3). Fibroblast-like synoviocytes (FLS), as the main constituent cell type of TGCT, play an important role in its proliferation, recurrence, and malignant transformation (4). Because its pathogenesis is currently unclear, the therapeutic mainstay of TGCT is limited to removing the entire pathological synovial tissue (5). However, surgical resection alone often cannot completely remove the diseased synovium; the residual synovium will rapidly proliferate and cause postoperative recurrence, at a rate of approximately $21-50 \%$ (6). Postoperative radiotherapy is often needed to reduce recurrence, but radiotherapy can lead to a series of complications such as joint adhesion (7). Therefore, there is an urgent need to explore the pathogenesis of TGCT and identify novel therapeutic targets, especially focusing on the inhibition of its proliferation and recurrence.

$\beta$-Arrestin 2 (Arrb2) is widely expressed in various tissues and involved in mediating $G$ protein coupled receptor (GPCR) desensitization, internalization, degradation and 
recycling (8). Recent studies have shown that Arrb2 is not only a signal terminator, but also a scaffold protein for GPCRs to mediate pathway signaling (9). It can enter the nucleus and, directly or indirectly, regulate the transcription factors and gene expression (10). Studies have reported that it plays a regulatory role in tumor occurrence, invasion and metastasis, in lung (11), ovarian (12), and liver cancer (13). However, it remains unclear whether Arrb2 is involved in the pathogenesis of TGCT and related to the high proliferation and recurrence ability of TGCT FLS. In this regard, we used mRNA-seq to compare TGCT and osteoarthritis (OA) synovial tissue by high-throughput sequencing, aiming to explore the molecular mechanisms of TGCT high proliferation and recurrence.

\section{Materials and Methods}

Patient samples. A total of 21 and 20 synovial tissues were collected from TGCT and OA patients, respectively, under protocols approved by the Ethics Committee of Peking University Third Hospital. All patients had signed an informed consent form. TGCT synovial tissues were derived from patients who had undergone an arthroscopic synovectomy, while OA synovial tissues were obtained at the time of total knee replacement. All synovial tissues were used for further FLS culture and histological testing. The clinical characteristics of these patients are listed in Table I.

Histological assessment. TGCT and OA patient's synovial tissues were excised and fixed in $4 \%$ paraformaldehyde for $48 \mathrm{~h}$, dehydrated in a series of ethanol, and embedded in paraffin. The samples were cut into $6-\mu \mathrm{m}$ thick sections and stained with hematoxylin-eosin $(\mathrm{H} \& \mathrm{E})$. For immunohistochemistry (IHC), the paraffin sections were incubated with $3 \% \mathrm{H}_{2} \mathrm{O}_{2}$ for $15 \mathrm{~min}$ following by incubation with pepsin for $30 \mathrm{~min}$ at $37^{\circ} \mathrm{C}$ for nonspecific antigen blocking. The sections were then incubated with antibodies against Ki-67 (Abcam, Cambridge, MA, USA; 1:500) and CD163 (Abcam, Cambridge, MA, USA; 1:200) overnight at $4^{\circ} \mathrm{C}$. Samples were then incubated with horseradish peroxidaseconjugated anti-mouse or anti-rabbit secondary antibodies (Zsjqbio, Beijing, PR China) at room temperature for $1 \mathrm{~h}$ before adding 3,3' diaminobenzidine (DAB) for visualization.

$m R N A$-seq array analysis. Total RNA was extracted from 3 TGCT and 3 OA synovial tissues using Trizol reagent (Invitrogen, Carlsbad, CA, USA). Microarray analysis was performed and analyzed by a commercial company (NovelBio Bio-Pharm Technology Co.,Ltd, Beijing, China) using the VAHTSTM Total RNA-seq (H/M/R) Library Prep Kit. Differential gene and transcript expression analysis of RNA-seq were examined using the TopHat and Cufflinks algorithms (14). Significant analysis was performed using the $p$-value and false discovery rate (FDR) analysis (15). At the same time, differentially expressed genes were identified with: fold change $>2$ or fold change $<0.5$, FDR $<0.05$. Pathway analysis was used to identify the significantly affected pathways of the differentially expressed genes, according to the Kyoto Encyclopedia of Genes and Genomes (KEGG) database (16). The threshold of significance was defined by $p$-value (17). Pathway activity network was constructed using Cytoscape (18) for graphical representations
Table I. Clinical characteristics of TGCT and OA patients.

\begin{tabular}{lcc}
\hline Characteristics & TGCT (n) & OA (n) \\
\hline Patients & 21 & 20 \\
Gender & 8 & 17 \\
$\quad$ Male & 13 & 3 \\
Female & $41.7 \pm 9.2$ & $58.5 .2 \pm 7.5$ \\
Age* & 18 & \\
Location & 2 & 0 \\
Knee & 1 & 0 \\
Elbow & & \\
Ankle & & \\
\hline
\end{tabular}

TGCT, Tenosynovial giant cell tumor; OA, osteoarthritis. *Data presented as mean \pm standard deviation.

of enriched biological pathways with significance $(p<0.05)$, including up-regulated and down-regulated ones.

Cell isolation and culture. FLS were isolated using enzymatic dispersion of the synovial tissue from TGCT and OA patients. Briefly, the synovial tissues were minced and incubated with $1 \mathrm{mg} / \mathrm{ml}$ type I collagenase (Invitrogen, Carlsbad, CA, USA) in low-glucose Dulbecco's modified Eagle's medium (DMEM Gibco, Carlsbad, CA, USA) for $2-3 \mathrm{~h}$ at $37^{\circ} \mathrm{C}$. Then, the cell suspensions were placed in culture dishes with DMEM supplemented with $10 \%$ (volume/volume, $\mathrm{v} / \mathrm{v}$ ) fetal bovine serum (Invitrogen), $100 \mathrm{U} / \mathrm{ml}$ penicillin, and 100 $\mu \mathrm{g} / \mathrm{ml}$ streptomycin, in a $37^{\circ} \mathrm{C}, 5 \% \mathrm{CO}_{2}$ incubator. After the third passage, non-FLS cells were completely depleted from these culture systems and the remaining cells were primarily FLS.

Cell transfection. Arrb2 small interfering RNA (siRNA) and scrambled negative control siRNA were obtained from Genechem Co. Ltd. (Shanghai, PR China). FLS were transfected with Arrb2 siRNA and scrambled negative control siRNA via Lipofectamine Max (Invitrogen, Carlsbad, CA, USA) according to the manufacturer's instructions. The final concentration of siRNA was $20 \mathrm{nM}$. The Arrb2 overexpression adenovirus (Ad-Arrb2) and negative control adenovirus (Ad- $\beta$-gal) were also obtained from Genechem Co. Ltd. FLS were transfected with control adenovirus (Ad- $\beta$-gal) and Ad-Arrb2 designed to overexpress Arrb2. The final multiplicity of infection (MOI) was 20 .

Western blot. TGCT and OA FLS were lysed in radioimmunoprecipitation assay (RIPA) lysis buffer, separated by SDS polyacrylamide gel electrophoresis (PAGE) and transferred to a polyvinylidene fluoride (PVDF) membrane. The membranes were then incubated with corresponding primary antibodies overnight at $4{ }^{\circ} \mathrm{C}$, followed by the corresponding secondary antibodies $(1 \mathrm{~h}$ at room temperature), and visualized using the BIO-RAD ChemiDoc XRS + system (Bio-Rad Laboratories, Hercules, CA, USA). The antibody against Arrb2 (Cat\#: sc13140; 1:1,000 dilution) was from Santa Cruz (Delaware, CA, USA); antibodies against phospho-ERK (Cat\#: cst. 4370; 1:1,000 dilution), total-ERK (Cat\#: cst 4695; 1:1,000 dilution), phospho-Akt (Cat\#: cst 4060s; 1:1,000 dilution) and total-Akt (Cat\#: cst 9272s; 1:1,000 dilution) were from Cell Signaling Technology (Boston, MA, USA); antibody against Arrb1 (Cat\#: ab32099-100; 1:2,000 dilution) was from Abcam (Cambridge, UK) and to 
glyceraldehyde-3-phosphate dehydrogenase (GAPDH) from EasyBio. (Cat\#: BE0023; 1:2,500 dilution, Beijing, PR China). The cells lines used in each test were obtained from five different patients, and the experiment used five biological replicates; each biological sample tests was performed in triplicate.

MTT assay. The cell proliferation of TGCT or OA FLS was evaluated by MTT assay. FLS were maintained in 96-well plates with 3,000 cells/well. MTT solution (Sigma, Saint Louis, MO, USA) was added to each well at day $0,2,4$ and 6 of the culture periods. After $4 \mathrm{~h}$ of incubation at $37^{\circ} \mathrm{C}$, the supernatant was removed, and $200 \mathrm{ml}$ dimethyl sulfoxide was added to dissolve the precipitate. Absorbance was examined at $450 \mathrm{~nm}$ using a Multiskan plate reader (Thermo Fisher Scientific, Boston, MA, USA). Each measurement was repeated three times.

Relative caspase 3 activity assay. Caspase 3 activity detection of TGCT and OA FLS was performed using the caspase 3 Activity Kit (Solarbio, Beijing, PR China). After 6 days of culture treated with Ad-Arrb2, Arrb2 siRNA, or Akt inhibitor (LY294002, Cat\#: 9901, CST, Boston, MA, USA), total protein was extracted from FLS and incubated with $90 \mu \mathrm{l}$ of prepared reaction buffer and $10 \mu \mathrm{l}$ caspase3 substrate (Ac-DEVD-pNA, $2 \mathrm{mM}$ ), in 96 -well plates at $37^{\circ} \mathrm{C}$ for $4 \mathrm{~h}$ in the dark. Then, the reaction mixtures were quantified at a wavelength of $405 \mathrm{~nm}$ using a Multiskan plate reader (Thermo Fisher Scientific). Each measurement was performed in triplicate.

Statistical analysis. All statistical analyses were performed using SPSS 20.0 (IBM Corp, Chicago, USA). Data were presented as mean \pm standard deviation. Statistical comparisons of two independent groups were performed using the Student's $t$-test. Multiple comparisons were performed using one-way ANOVA. Statistically significant data are indicated by $* p<0.05$, ** $p<0.01$, and $* * * p<0.001$.

\section{Results}

Arrb2 is highly expressed in TGCT FLS. In the present study, we first confirmed the general pathological features of TGCT via arthroscopy and MRI and compared them with OA. Under arthroscopy, the TGCT synovium was significantly hyperplastic compared to the OA synovium, and it was reddish brown due to hemosiderin. MRI showed that the synovial tissue of TGCT was widely diffused in the joint cavity, and caused severe joint edema (Figure 1A). Furthermore, H\&E staining showed that the TGCT synovial lining layer was thickened. The immunohistochemical analysis revealed that the expression of Ki-67 and CD 163 was significantly up-regulated compared to OA (Figure 1B). To determine whether there are differences in the levels of mRNA expression between TGCT and OA, we used mRNAseq array and identified 1,314 mRNAs that were significantly different; $780 \mathrm{mRNAs}$ were down-regulated and 534 mRNAs were up-regulated. Among them, we found that Arrb2 was significantly up-regulated in TGCT, compared to the OA (Figure 1C), and the expression of Arrb2 was closely related to the regulation of cell proliferation (Figure 1D). Next, we used western bolt to verify the protein levels of
Arrb2 and Arrb1 in tissue samples and cell samples from TGCT and OA patients. The results showed that Arrb2 was highly expressed in both TGCT tissue (Figure 1E) and FLS (Figure 1F), compared to OA-derived samples, while there was no significant difference in Arrb1 expression; thus, confirming the mRNA-seq array results. Hence, we focused on Arrb2 for further study.

Arrb2 facilitates the proliferation and inhibits the apoptosis of TGCT FLS. We first examined the proliferation ability of TGCT and OA FLS by MTT assay. The results showed that the proliferation ability of TGCT FLS was significantly stronger than that of OA FLS. At the same time, the caspase 3 activity assay showed that, TGCT FLS apoptosis was lower than that of OA FLS (Figure 2A). Overexpression of FLS Arrb2 by AdArrb2 at MOI 20 showed significant up-regulation of Arrb2 (Figure 2B) compared to the negative control (Ad- $\beta$-gal), while overexpression of Arrb2 did not affect the expression of Arrb1 (Figure 2C). Subsequently, we tested the proliferation and apoptosis of transfected TGCT and OA FLS to overexpress Arrb2. The results demonstrated that following overexpression, regardless of TGCT or OA, the proliferation ability of FLS was enhanced and apoptosis was inhibited, compared to the negative control cells (Figure 2D and E).

Arrb2 knockdown inhibits the proliferation and increases apoptosis of TGCT FLS. We then constructed Arrb2 siRNA to transfect FLS. Western blot analysis confirmed that Arrb2 siRNA effectively knocked down the expression of Arrb2, without affecting Arrb1 expression (Figure 3A and B). Moreover, we analyzed the effects on cell proliferation ability and apoptosis after silencing Arrb2, using the MTT and caspase-3 assays. We confirmed that silenced Arrb2 inhibited the proliferation and induced the apoptosis of both TGCT and OA FLS, compared to the negative control (Figure 3C and D).

Arrb2 regulates the TGCT FLS proliferation and apoptosis by regulating the PI3K-Akt cell survival pathway. We next investigated the mechanism underlying the effects of Arrb2 on the cell proliferation ability and apoptosis. The KEGG pathway analysis results showed that, when compared to OA, the activation of multiple pathways in TGCT had changed, including TGF- $\beta$ signaling pathway, Rap 1 signaling pathway, PPAR signaling pathway and PI3K-Akt signaling pathway. Among them, PI3K-Akt, as a pathway related to cell survival, was significantly activated (Figure 4A). In order to verify the KEGG results, western bolt analysis was performed. The results confirmed that the phosphorylation levels of Akt and Erk1/2 were significantly higher in TGCT synovial tissue or FLS than in OA (Figure 4B).

To further examine the role of Arrb2 in the PI3K-Akt pathway in FLS, we treated the cultured TGCT and OA FLS 
A

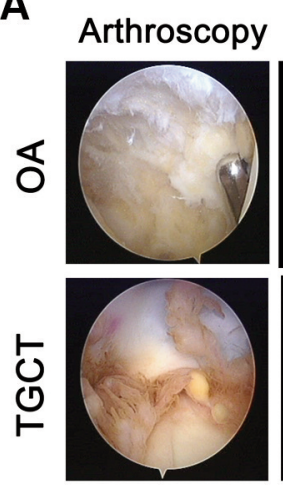

C

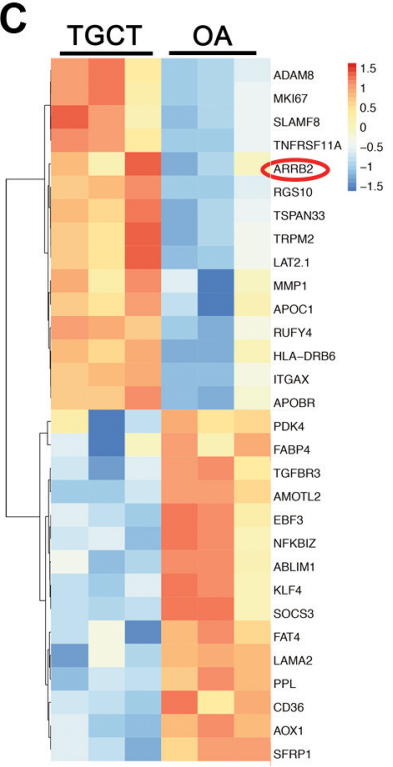

B

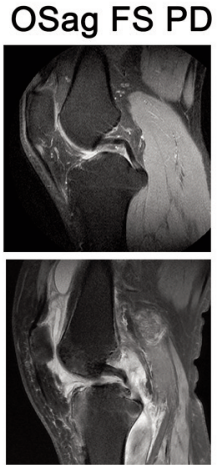

D

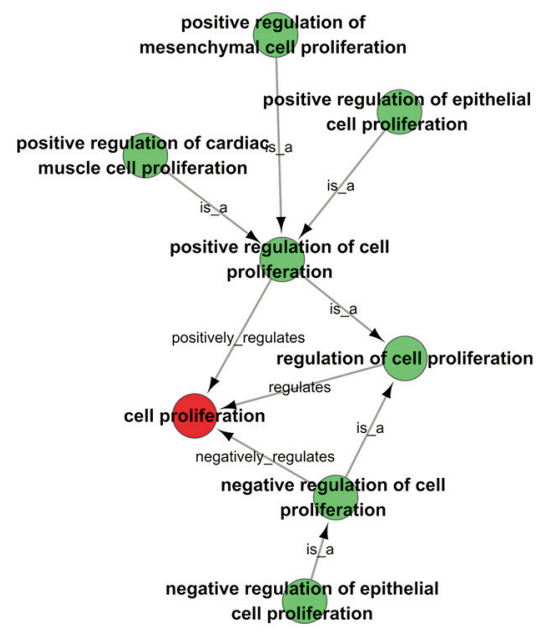

$H \& E$
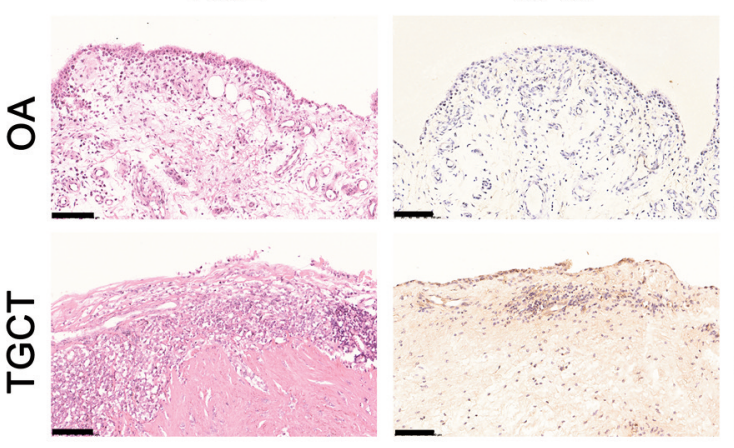

E

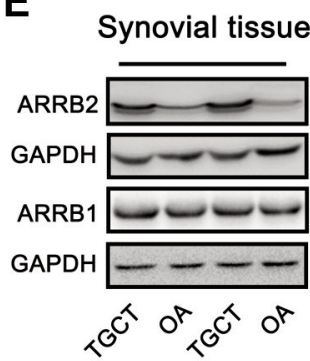

$\mathbf{F}$

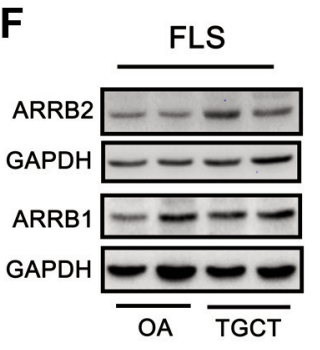

CD163
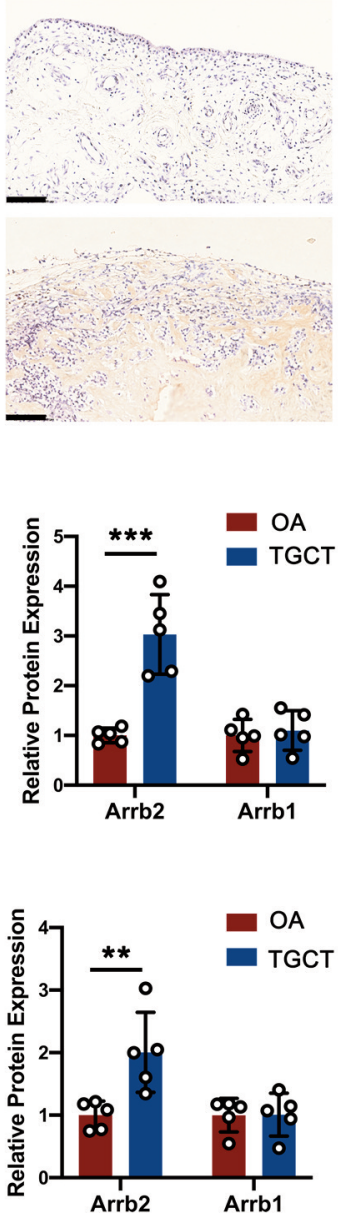

Figure 1. Arrb2 is up-regulated in TGCT patients compared with OA. (A) Comparison of the characteristics of TGCT and OA synovitis under arthroscopy and MRI. (B) Comparison of the pathological features of TGCT and OA synovitis using H\&E and IHC; Scale bar: $100 \mu$ m. (C) Heatmap of the differentially expressed mRNA patterns in TGCT versus OA synovitis, the relative expression is indicated by color scale (red: high expression). (D) Gene ontology analysis between TGCT and OA synovitis (green: up regulation, red: down regulation). (E-F) Western blot verifies the expression of Arrb2 and Arrb1 in TGCT and OA synovitis tissues (E) and FLS (F). TGCT, Tenosynovial giant cell tumor; OA, osteoarthritis; FLS, fibroblast-like synoviocytes; OSag FS PD, oblique sagittal fat suppressed-proton density imaging; H\&E, hematoxylin-eosin staining; GAPDH, glyceraldehyde-3-phosphate dehydrogenase. $* p<0.05 ; * * p<0.01 ; * * * 00.001$.

with Ad-Arrb2. After overexpressing Arrb2, the phosphorylation levels of Akt and Erk1/2 in both TGCT and OA FLS were significantly higher than that of the negative controls, indicating that Arrb2 can effectively activate this pathway (Figure 4C). Furthermore, inhibition of the PI3KAkt pathway with Akt inhibitor in Ad-Arrb2-transfected TGCT FLS inverted the effects of Arrb2 overexpression on cell proliferation and apoptosis (Figure 4D). Next, using western blot, we analyzed the phosphorylation levels of PI3K-Akt pathway after Arrb2 silencing. The results showed that after treatment of TGCT and OA FLS with Arrb2 siRNA, the phosphorylation levels of Akt and Erk1/2 were significantly down-regulated, compared to the negative controls (Figure 4E and F). Taken together, although the PI3K-Akt pathway may not be the only pathway regulated by Arrb2 in TGCT (other pathways could also be involved), we conclude that Arrb2 positively regulates survival and negatively regulates apoptosis of TGCT and OA FLS, at least partially, by activating the PI3K-Akt pathway.

\section{Discussion}

TGCT, also known as pigmented villonodular synovitis (PVNS), is characterized by rapid proliferation and high 

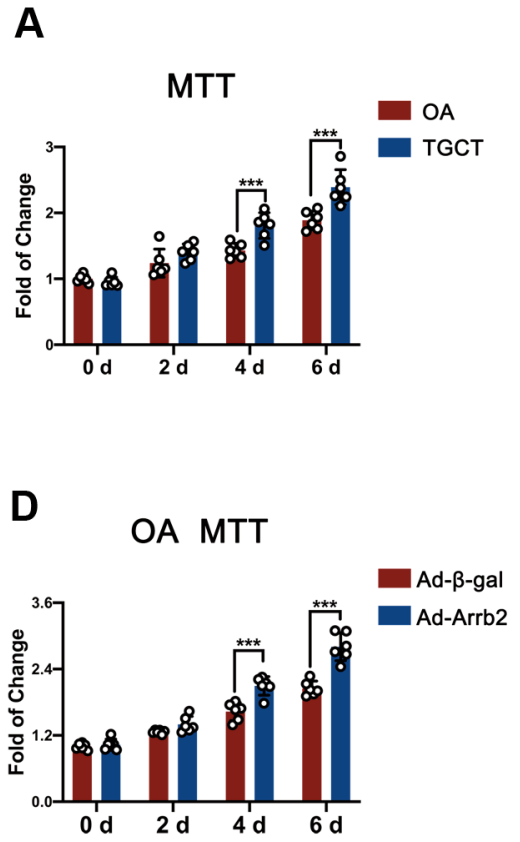

Caspase 3

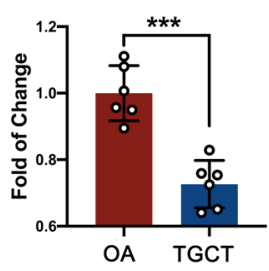

OA Caspase 3

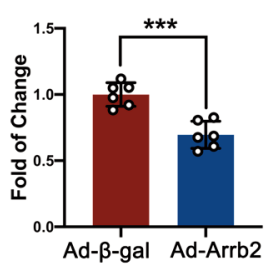

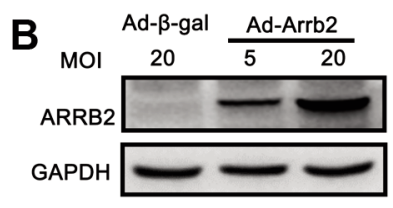

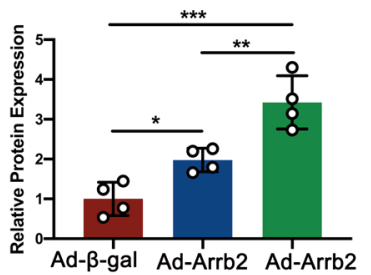

E

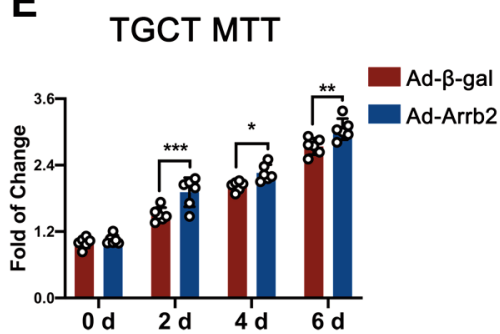

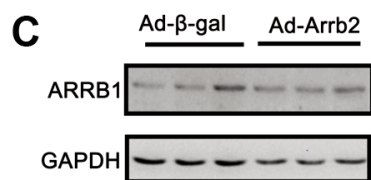

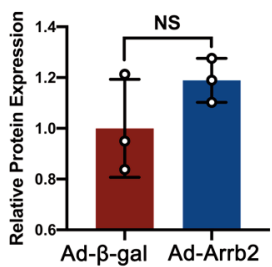

TGCT Caspase 3

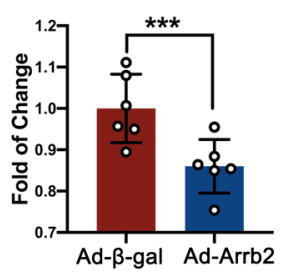

Figure 2. Arrb2 facilitates the proliferation and inhibits the apoptosis of TGCT FLS. (A) MTT and caspase 3 activity assay of TGCT and OA. (B) Western blot showing the overexpression ability of Arrb2 adenovirus vector. (C) Western blot showing the effect of Arrb2 adenovirus vector on Arrb1 expression. (D) MTT and caspase 3 activity assay of OA FLS treated with or without Arrb2 adenovirus vector. (E) MTT and caspase 3 activity assay of TGCT FLS treated with or without Arrb2 adenovirus vector. TGCT, Tenosynovial giant cell tumor; OA, osteoarthritis; FLS, fibroblast-like synoviocytes; MTT, 3-(4,5)-dimethylthiahiazo (-z-y1)-3,5-di-phenytetrazoliumromide; MOI, multiplicity of infection; GAPDH, glyceraldehyde-3phosphate dehydrogenase. $* p<0.05 ; * * p<0.01 ; * * * p<0.001$.

recurrence rate and its pathogenesis is currently unclear to clinicians (19). Due to the incomplete research on the pathogenesis of TGCT, a complete surgical synovectomy remains the principle treatment; however there is no generally accepted target about the surgical procedure and the postoperative adjunctive therapy (20). Therefore, further understanding of TGCT pathogenesis and an exploration of novel therapeutic targets for patients is urgently required.

TGCT as well as other types of synovitis are very different in terms of pathological characteristics. Herein, we showed that under arthroscopy, the TGCT synovial tissue was obviously hyperplastic and was reddish brown due to the deposition of hemoglobin. Immunohistochemical analysis demonstrated that the expression of the proliferation marker $\mathrm{Ki}-67$ and the macrophage marker CD163 was higher in TGCT than in OA synovial tissue.

In order to explore the pathogenesis of TGCT and find new therapeutic targets, we used an mRNA-seq highthroughput assay to screen the differentially expressed genes between TGCT and OA synovial tissue. The results showed that 780 mRNAs were down-regulated and 534 were upregulated. Among them, Arrb2 was significantly up-regulated in TGCT compared to OA, and the expression of Arrb2 was closely related to the regulation of cell proliferation. Arrb2 is the main member of the arrestin family in non-retinal expression (21). It is generally known that $\beta$-arrestin modulates GPCR signaling through receptor desensitization and internalization (22). Many recent studies have shown that $\beta$-arrestin unexpectedly acts as scaffold protein for many signal molecules in the cytoplasm and nucleus, thereby regulating gene expression and cell responses (23-25). There is increasing evidence that abnormal $\beta$-arrestin expression is involved in several types of tumors (26) as well as being related to cell survival (27), apoptosis (28), migration (29) and proliferation (26). Jude Masannat et al. found that knockout of Arrb2 decreases the proliferation and migration rates of renal cell carcinoma, in vitro, and xenograft tumor growth in animals (30). Similarly, Fanling Hong et al. showed that Arrb2 overexpression promoted the growth of human endometrial carcinoma heterotransplants and inhibited the endometrial cell apoptosis (31). On the other hand, Yimei Wang et al. indicated that Arrb2 promotes cell death and inhibits cell pro-survival signaling (32). These discrepancies may be due to the distinct Arrb2 conformational states triggered by different upstream stimuli and different interacting proteins in different tissues. 

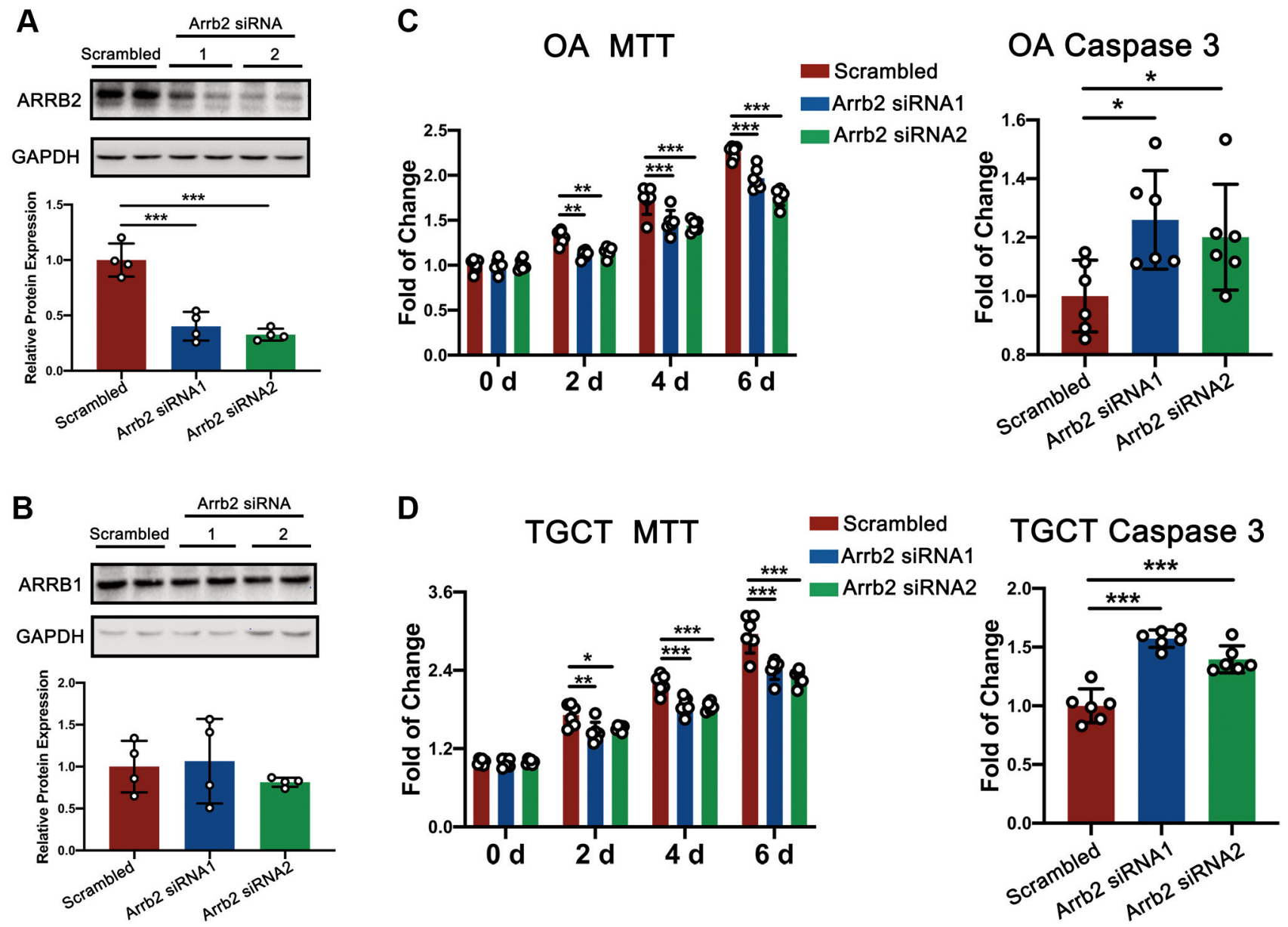

Figure 3. Knockdown the Arrb2 inhibits the proliferation and increases the apoptosis of TGCT FLS. (A) Western blot showing the knockdown ability of Arrb2 siRNA. (B) Western blot showing the effect of Arrb2 siRNA on Arrb1 expression. (C) MTT and caspase 3 activity assay of OA FLS treated with or without Arrb2 siRNA. (D) MTT and caspase 3 activity assay of TGCT FLS treated with or without Arrb2 siRNA. TGCT, Tenosynovial giant cell tumor; OA, osteoarthritis; MTT: 3-(4,5)-dimethylthiahiazo (-z-y1)-3,5-di-phenytetrazoliumromide; GAPDH: glyceraldehyde-3-phosphate dehydrogenase. $* p<0.05 ; * * p<0.01 ; * * * p<0.001$.

However, there is no report on its role in TGCT. Our western bolt results confirmed that whether it is synovial tissue or FLS, the expression of TGCT Arrb2 was higher than that of OA. However, some studies have shown that the expression of Arrb1 is also essential for maintaining the malignant phenotype of tumors (33-35). Therefore, we also tested the expression of Arrb1 and found that there was no difference between TGCT and OA.

Subsequently, we tested the proliferation and apoptosis of TGCT and OA FLS and found that the proliferation ability was higher and apoptosis was lower in TGCT than in OA FLS. To further explore the effect of Arrb2 on FLS proliferation and apoptosis, we constructed an Arrb2 overexpression adenovirus vector and Arrb2 siRNA to interfere with the expression Arrb2 in FLS. The results confirmed that when Arrb2 overexpression in FLS significantly promoted cell proliferation and inhibited cell apoptosis. On the contrary, when Arrb2 was knocked down, cell proliferation was inhibited and apoptosis returned to normal levels. Interestingly, this phenomenon not only occurred in the TGCT FLS, but also in the OA FLS, indicating that Arrb2 can not only regulate cells with high proliferation characteristics, but also cells with relatively weak proliferative ability. A previous study has also shown that Arrb2 is positively correlated with human FLS proliferation (36), which is consistent with our research.

The molecular mechanism by which Arrb2 regulates the proliferation and apoptosis of TGCT FLS was also explored in this study. The KEGG pathway analysis results showed that, compared with to OA, multiple pathways were significantly activated in TGCT. Among them, the PI3K-Akt pathway is widely reported to be related to cell survival and 
A

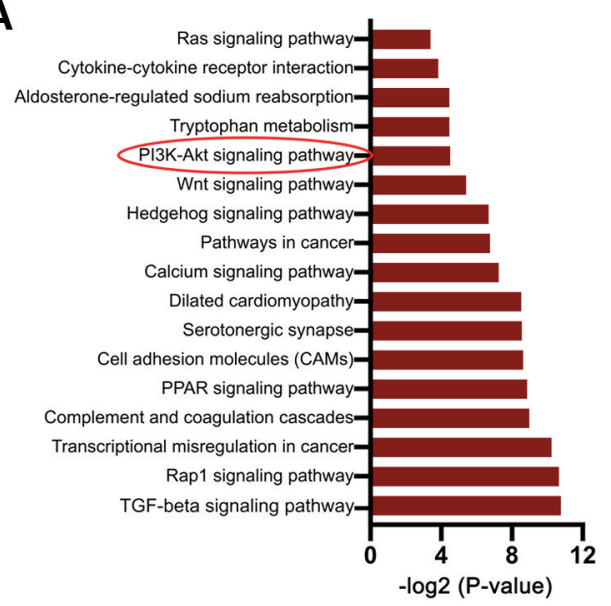

B

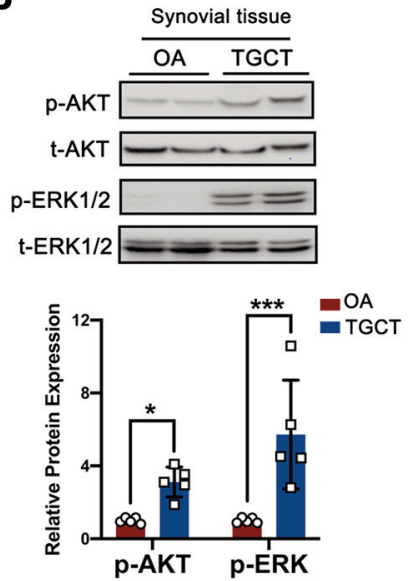

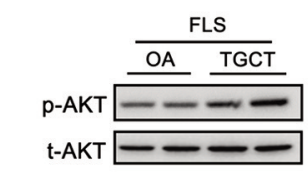

p-ERK $1 / 2 \equiv=\equiv$

t-ERK $1 / 2=-=-$

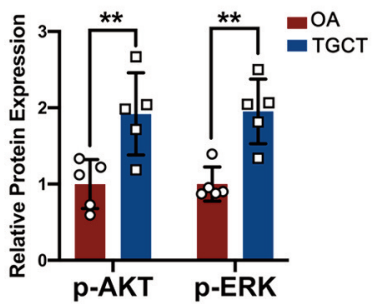

C
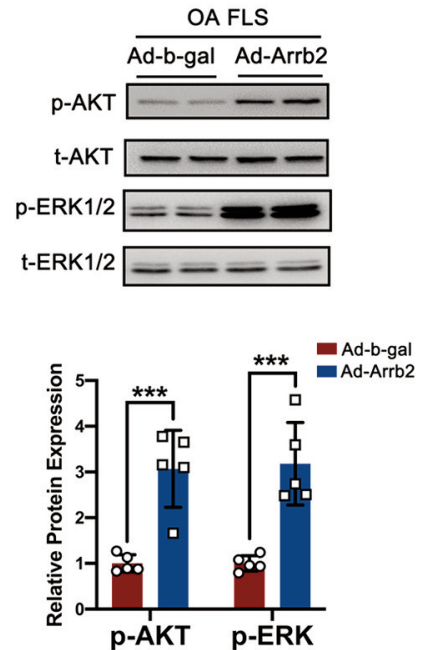

D

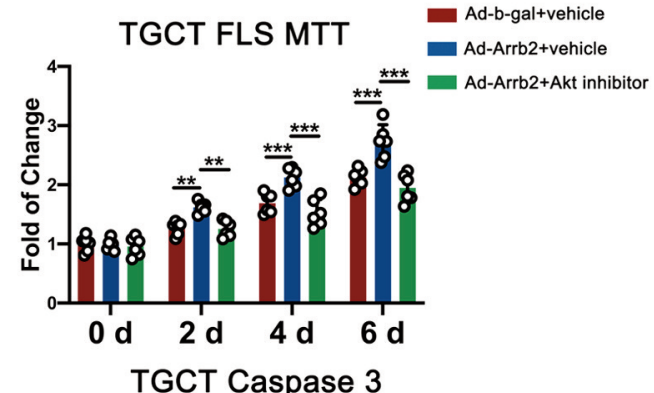

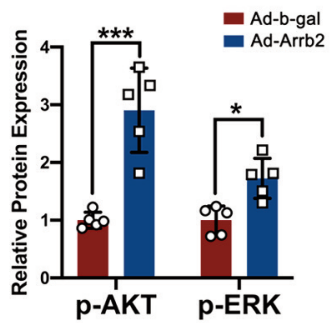
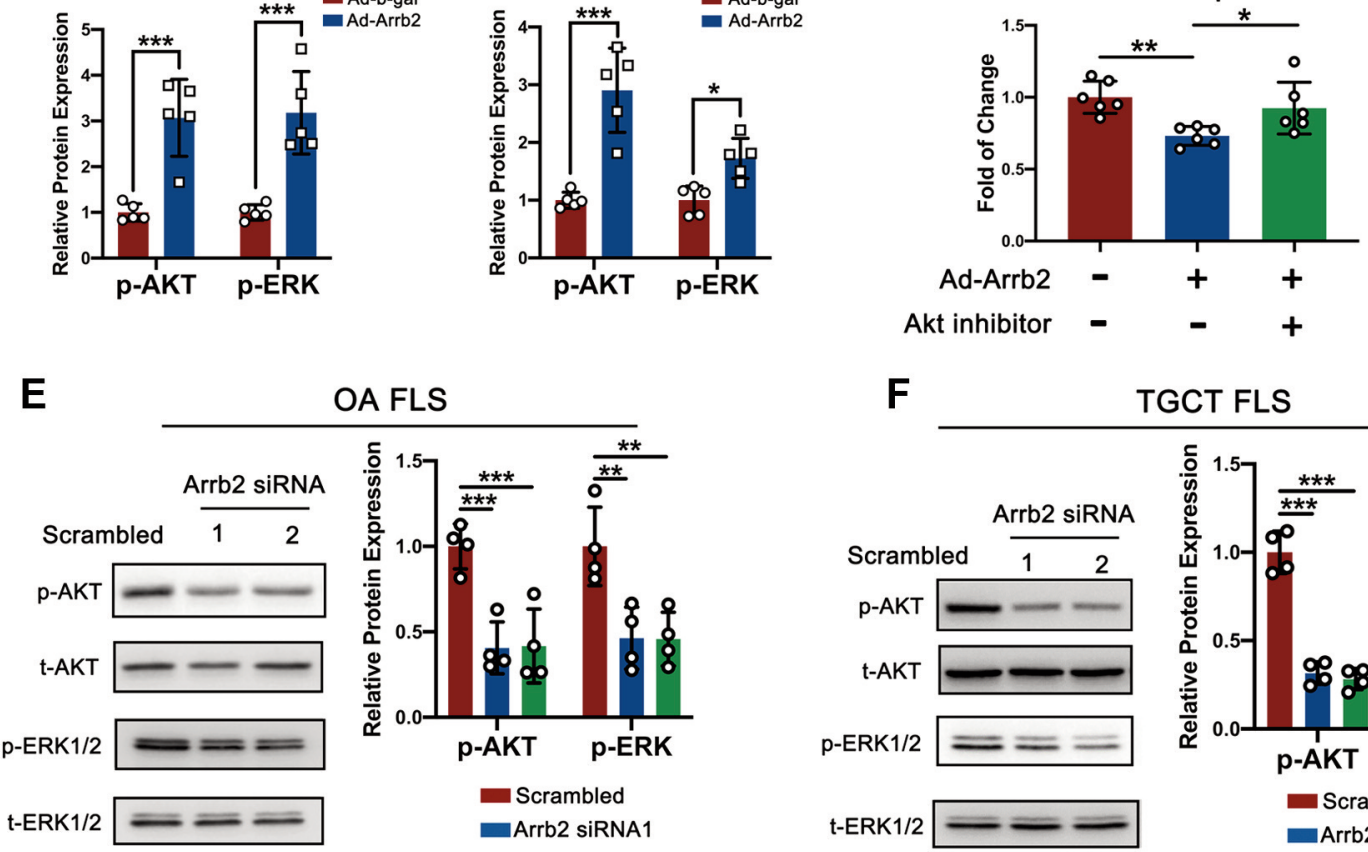

OA FLS
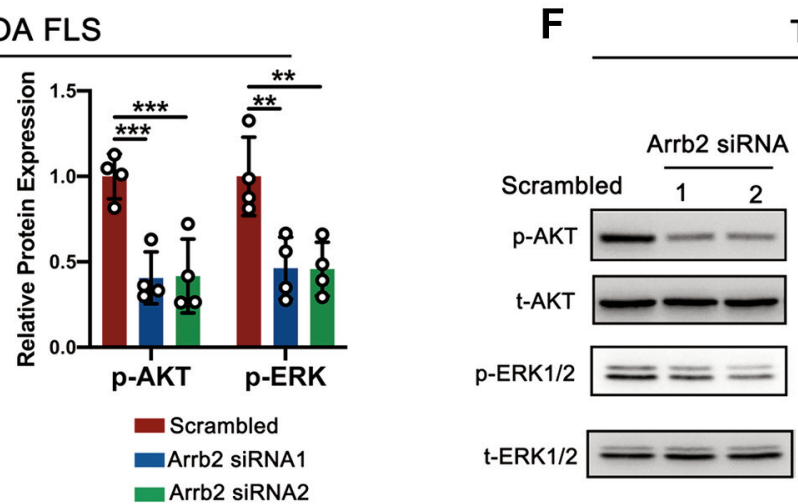

TGCT FLS

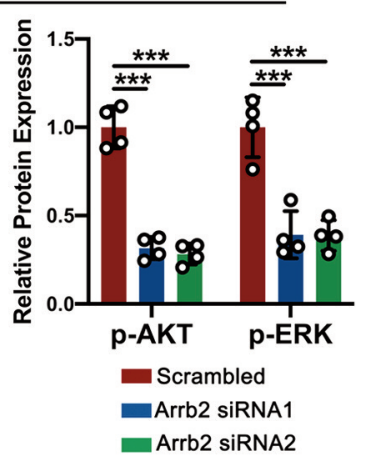

Figure 4. Arrb2 regulates the TGCT FLS proliferation and apoptosis by regulating the PI3K-Akt cell survival pathway. (A) The KEGG pathway analysis of mRNA-seq results between TGCT and OA synovitis. (B) Western blot showing the phosphorylation levels of Akt and Erk1/2 in TGCT and OA synovitis tissues and FLS. (C) Western blot showing the phosphorylation levels of Akt and Erk1/2 in TGCT and OA FLS treated with or without Arrb2 adenovirus vector. (D) MTT and caspase 3 activity assay of TGCT FLS treated with or without Arrb2 adenovirus vector and Akt inhibitor. (E-F) Western blot showing the phosphorylation levels of Akt and Erk1/2 in TGCT and OA FLS treated with or without Arrb2 siRNA. TGCT, Tenosynovial giant cell tumor; OA, osteoarthritis; MTT, 3-(4,5)-dimethylthiahiazo (-z-y1)-3,5-di-phenytetrazoliumromide; GAPDH, glyceraldehyde-3-phosphate dehydrogenase. ${ }^{*} p<0.05 ; * * p<0.01 ; * * p<0.001$. 
proliferation $(37,38)$, especially in tumors $(39,40)$. Furthermore, the PI3K-Akt pathway plays an important role in synovitis; our previous research has confirmed that inflammatory factors can regulate the invasion and migration of OA FLS through the PI3K-Akt pathway (41). At the same time, Arrb2 can participate in several types of cancers by acting as a scaffold protein in various signaling pathways, including extracellular regulatory protein kinase (ERK1/2), $\mathrm{Wnt} / \beta$-catenin and c-Src (9). Previous research has also shown that $\beta$-arrestins are involved in cell proliferation, apoptosis, and differentiation by mediating the Akt phosphorylation response to extensive stimulation in different cell types $(42,43)$. Furthermore, PI3K-Akt signaling has been shown to play a central role in Arrb2medited cell protection $(44,45)$. Therefore, we tested whether PI3K-Akt pathway is the downstream signaling mediator of the Arrb2-induced anti-apoptotic and proproliferation effects in TGCT. We first compared the phosphorylation levels of Akt and ERK1/2 in TGCT and OA, demonstrating that whether it is synovial tissue or FLS, phosphorylation levels of these proteins were significantly higher in TGCT than in OA. When Arrb2 was overexpressed or knocked down, the phosphorylation levels of Akt and ERK1/2 in FLS were significantly increased or decreased, respectively (Figure 4C, E and F), suggesting that Arrb2 can effectively regulate the activation of this pathway. Most importantly, Akt inhibitor inverted the effects of Arrb2 overexpression on cell proliferation and apoptosis of TGCT FLS. The above results strongly suggest that Arrb2 regulates the TGCT FLS proliferation and apoptosis mainly through the PI3K-Akt cell survival pathway.

In conclusion, this is the first detailed study to explore whether and how Arrb2 affects TGCT FLS proliferation and apoptosis. Our findings demonstrated that Arrb2 facilitates the proliferation and inhibits the apoptosis of TGCT FLS mainly through PI3K-Akt activation, providing new insights into the molecular mechanism of TGCT. In this regard, Arrb2 may have the potential to be a molecular target for TGCT therapy.

\section{Conflicts of Interests}

The Authors declare that they have no competing interests.

\section{Authors' Contributions}

CXC conducted the majority of the experiments and completed the manuscript; YZ participated in the experiment and helped with manuscript preparation; YFA and XZ conceived the project and designed the experiments; JC and FW collected the clinical specimens; XYN and XQH revised the manuscript; XF, XND and JYZ analyzed the data, prepared the draft figures; YFA and XZ supervised the project; CXC and YZ contributed equally in this work. All Authors approved the final article.

\section{Acknowledgements}

This work was supported by grants from the National Natural Science Foundation of China (Grant No. 8187090401), Beijing Natural Science Foundation (Grant No. 7192221), and the Clinical Medicine Plus X - Young Scholars Project, Peking University (Grant No. BMU2019LCKXJ008 and PKU2020LCXQ012), the Fundamental Research Funds for the Central Universities.

\section{References}

1 Willimon SC, Busch MT and Perkins CA: Pigmented villonodular synovitis of the knee: An underappreciated source of pain in children and adolescents. J Pediatr Orthop 38(8): e482-e485, 2018. PMID: 29917011. DOI: 10.1097/BPO.0000000000001213

2 Gouin F: Nilotinib in locally advanced pigmented villonodular synovitis: challenges of a new targeted therapy. Lancet Oncol 19(5): 584-586, 2018. PMID: 29571947. DOI: 10.1016/S14702045(18)30199-2

3 Imakiire N, Fujino T, Morii T, Honya K, Mochizuki K, Satomi $\mathrm{K}$ and Fujioka Y: Malignant pigmented villonodular synovitis in the knee - report of a case with rapid clinical progression. Open Orthop J 5: 13-16, 2011. PMID: 21270951. DOI: 10.2174/ 1874325001105010013

4 Byers PD, Cotton RE, Deacon OW, Lowy M, Newman PH, Sissons HA and Thomson AD: The diagnosis and treatment of pigmented villonodular synovitis. J Bone Joint Surg Br 50(2): 290-305, 1968. PMID: 4297226.

5 Bernthal NM, Ishmael CR and Burke ZDC: Management of pigmented villonodular synovitis (PVNS): an orthopedic surgeon's perspective. Curr Oncol Rep 22(6): 63, 2020. PMID: 32494892. DOI: 10.1007/s11912-020-00926-7

6 Palmerini E, Staals EL, Maki RG, Pengo S, Cioffi A, Gambarotti M, Picci P, Daolio PA, Parafioriti A, Morris C, Antonescu CR, Gronchi A, Casali PG, Donati DM, Ferrari S and Stacchiotti S: Tenosynovial giant cell tumour/pigmented villonodular synovitis: outcome of 294 patients before the era of kinase inhibitors. Eur J Cancer 51(2): 210-217, 2015. PMID: 25465190. DOI: $10.1016 /$ j.ejca.2014.11.001

7 Gouin $\mathrm{F}$ and Noailles T: Localized and diffuse forms of tenosynovial giant cell tumor (formerly giant cell tumor of the tendon sheath and pigmented villonodular synovitis). Orthop Traumatol Surg Res 103(1S): S91-S97, 2017. PMID: 28057477. DOI: $10.1016 /$ j.otsr.2016.11.002

8 Kang DS, Tian X and Benovic JL: Role of $\beta$-arrestins and arrestin domain-containing proteins in $\mathrm{G}$ protein-coupled receptor trafficking. Curr Opin Cell Biol 27: 63-71, 2014. PMID: 24680432. DOI: 10.1016/j.ceb.2013.11.005

9 DeWire SM, Ahn S, Lefkowitz RJ and Shenoy SK: Beta-arrestins and cell signaling. Annu Rev Physiol 69: 483-510, 2007. PMID: 17305471. DOI: 10.1146/annurev.physiol.69.022405.154749

10 Mo W, Zhang L, Yang G, Zhai J, Hu Z, Chen Y, Chen X, Hui L, Huang R and $\mathrm{Hu}$ G: Nuclear beta-arrestin 1 functions as a scaffold for the dephosphorylation of STAT1 and moderates the antiviral activity of IFN-gamma. Mol Cell 31(5): 695-707, 2008. PMID: 18775329. DOI: 10.1016/j.molcel.2008.06.017

11 Xiao K, McClatchy DB, Shukla AK, Zhao Y, Chen M, Shenoy SK, Yates JR 3rd and Lefkowitz RJ: Functional specialization of beta-arrestin interactions revealed by proteomic analysis. Proc 
Natl Acad Sci USA 104(29): 12011-12016, 2007. PMID: 17620599. DOI: $10.1073 /$ pnas 0704849104

12 Rosanò L, Cianfrocca R, Masi S, Spinella F, Di Castro V, Biroccio A, Salvati E, Nicotra MR, Natali PG and Bagnato A: Beta-arrestin links endothelin A receptor to beta-catenin signaling to induce ovarian cancer cell invasion and metastasis. Proc Natl Acad Sci USA 106(8): 2806-2811, 2009. PMID: 19202075. DOI: $10.1073 /$ pnas 0807158106

13 Sun WY, Hu SS, Wu JJ, Huang Q, Ma Y, Wang QT, Chen JY and Wei W: Down-regulation of $\beta$-arrestin2 promotes tumour invasion and indicates poor prognosis of hepatocellular carcinoma. Sci Rep 6: 35609, 2016. PMID: 27759077. DOI: 10.1038/srep35609

14 Trapnell C, Roberts A, Goff L, Pertea G, Kim D, Kelley DR, Pimentel H, Salzberg SL, Rinn JL and Pachter L: Differential gene and transcript expression analysis of RNA-seq experiments with TopHat and Cufflinks. Nat Protoc 7(3): 562-578, 2012 PMID: 22383036. DOI: 10.1038/nprot.2012.016

15 Benjamini Y, Drai D, Elmer G, Kafkafi N and Golani I: Controlling the false discovery rate in behavior genetics research. Behav Brain Res 125(1-2): 279-284, 2001. PMID: 11682119. DOI: 10.1016/s0166-4328(01)00297-2

16 Kanehisa M and Goto S: KEGG: kyoto encyclopedia of genes and genomes. Nucleic Acids Res 28(1): 27-30, 2000. PMID: 10592173. DOI: $10.1093 / \mathrm{nar} / 28.1 .27$

17 Draghici S, Khatri P, Tarca AL, Amin K, Done A, Voichita C, Georgescu C and Romero R: A systems biology approach for pathway level analysis. Genome Res 17(10): 1537-1545, 2007. PMID: 17785539. DOI: 10.1101/gr.6202607

18 Shannon P, Markiel A, Ozier O, Baliga NS, Wang JT, Ramage D, Amin N, Schwikowski B and Ideker T: Cytoscape: a software environment for integrated models of biomolecular interaction networks. Genome Res 13(11): 2498-2504, 2003. PMID: 14597658. DOI: $10.1101 /$ gr.1239303

19 Jobe CM, Raza A and Zuckerman L: Pigmented villonodular synovitis: extrasynovial recurrence. Arthroscopy 27(10): 14491451, 2011. PMID: 21889289. DOI: 10.1016/j.arthro.2011.06.023

20 Ottaviani S, Ayral X, Dougados M and Gossec L: Pigmented villonodular synovitis: a retrospective single-center study of 122 cases and review of the literature. Semin Arthritis Rheum 40(6): 539546, 2011. PMID: 20884045. DOI: 10.1016/j.semarthrit.2010.07.005

21 Vishnivetskiy SA, Schubert C, Climaco GC, Gurevich YV, Velez MG and Gurevich VV: An additional phosphate-binding element in arrestin molecule. Implications for the mechanism of arrestin activation. J Biol Chem 275(52): 41049-41057, 2000. PMID: 11024026. DOI: $10.1074 /$ jbc.M007159200

22 Wong AM, Abrams MC and Micevych PE: $\beta$-arrestin regulates estradiol membrane-initiated signaling in hypothalamic neurons PLoS One 10(3): e0120530, 2015. PMID: 25803606. DOI: 10.1371/journal.pone.0120530

23 Monasky MM, Taglieri DM, Henze M, Warren CM, Utter MS, Soergel DG, Violin JD and Solaro RJ: The $\beta$-arrestin-biased ligand TRV120023 inhibits angiotensin II-induced cardiac hypertrophy while preserving enhanced myofilament response to calcium. Am J Physiol Heart Circ Physiol 305(6): H856-H866, 2013. PMID: 23873795. DOI: 10.1152/ajpheart.00327.2013

24 Eichel K, Jullié D, Barsi-Rhyne B, Latorraca NR, Masureel M, Sibarita JB, Dror RO and von Zastrow M: Catalytic activation of $\beta$-arrestin by GPCRs. Nature 557(7705): 381-386, 2018. PMID: 29720660. DOI: 10.1038/s41586-018-0079-1
25 de Waal PW, Shi J, You E, Wang X, Melcher K, Jiang Y, Xu HE and Dickson BM: Molecular mechanisms of fentanyl mediated $\beta$-arrestin biased signaling. PLoS Comput Biol 16(4): e1007394, 2020. PMID: 32275713. DOI: 10.1371/journal.pcbi.1007394

26 Raghuwanshi SK, Nasser MW, Chen X, Strieter RM and Richardson RM: Depletion of beta-arrestin-2 promotes tumor growth and angiogenesis in a murine model of lung cancer. $\mathrm{J}$ Immunol 180(8): 5699-5706, 2008. PMID: 18390755. DOI: 10.4049/jimmunol.180.8.5699

27 Dasgupta P, Rastogi S, Pillai S, Ordonez-Ercan D, Morris M, Haura E and Chellappan S: Nicotine induces cell proliferation by beta-arrestin-mediated activation of $\mathrm{Src}$ and Rb-Raf-1 pathways. J Clin Invest 116(8): 2208-2217, 2006. PMID: 16862215. DOI: $10.1172 / J C I 28164$

28 Ahn S, Kim J, Hara MR, Ren XR and Lefkowitz RJ: \{beta\}arrestin-2 mediates anti-apoptotic signaling through regulation of BAD phosphorylation. J Biol Chem 284(13): 8855-8865, 2009. PMID: 19171933. DOI: 10.1074/jbc.M808463200

29 Li TT, Alemayehu M, Aziziyeh AI, Pape C, Pampillo M, Postovit LM, Mills GB, Babwah AV and Bhattacharya M: Betaarrestin/Ral signaling regulates lysophosphatidic acid-mediated migration and invasion of human breast tumor cells. Mol Cancer Res 7(7): 1064-1077, 2009. PMID: 19609003. DOI: 10.1158/ 1541-7786.MCR-08-0578

30 Masannat J, Purayil HT, Zhang Y, Russin M, Mahmud I, Kim W, Liao D and Daaka Y: $\beta$ Arrestin2 mediates renal cell carcinoma tumor growth. Sci Rep 8(1): 4879, 2018. PMID: 29559707. DOI: $10.1038 / \mathrm{s} 41598-018-23212-\mathrm{w}$

31 Hong F, Zhang Y, Cheng W, Sun X and Wang J: $\beta$-arrestin-2 upregulates toll-like receptor 2 signaling and inhibits apoptosis in human endometrial cancer heterotransplants in nude mice. BMC Cancer 19(1): 1035, 2019. PMID: 31675995. DOI: 10.1186/ s12885-019-6254-4

32 Wang Y, Jin L, Song Y, Zhang M, Shan D, Liu Y, Fang M, Lv $\mathrm{F}$, Xiao RP and Zhang Y: $\beta$-arrestin 2 mediates cardiac ischemiareperfusion injury via inhibiting GPCR-independent cell survival signalling. Cardiovasc Res 113(13): 1615-1626, 2017. PMID: 29016703. DOI: $10.1093 / \mathrm{cvr} / \mathrm{cvx} 147$

33 Shu Y, Wang Y, Lv WQ, Peng DY, Li J, Zhang H, Jiang GJ, Yang BJ, Liu S, Zhang J, Chen YH, Tang S, Wan KX, Yuan JT, Guo W, Fu G, Qi XK, Liu ZD, Liu HY, Yang C, Zhang LH, Liu FJ, Yu J, Zhang PH, Qu B, Zhao H, He TC and Zou L: ARRB1-promoted NOTCH1 degradation is suppressed by OncomiR miR-223 in Tcell acute lymphoblastic leukemia. Cancer Res 80(5): 988-998, 2020. PMID: 31822496. DOI: 10.1158/0008-5472.CAN-19-1471

34 Son D, Kim Y, Lim S, Kang HG, Kim DH, Park JW, Cheong W, Kong HK, Han W, Park WY, Chun KH and Park JH: miR-374a$5 \mathrm{p}$ promotes tumor progression by targeting ARRB1 in triple negative breast cancer. Cancer Lett 454: 224-233, 2019. PMID: 31004703. DOI: 10.1016/j.canlet.2019.04.006

35 Dasgupta P, Rizwani W, Pillai S, Davis R, Banerjee S, Hug K, Lloyd M, Coppola D, Haura E and Chellappan SP: ARRB1mediated regulation of E2F target genes in nicotine-induced growth of lung tumors. J Natl Cancer Inst 103(4): 317-333, 2011. PMID: 21212384. DOI: 10.1093/jnci/djq541

36 Wu HX, Chen JY, Wang QT, Sun WY, Liu LH, Zhang LL and Wei W: Expression and function of $\beta$-arrestin 2 stimulated by IL-1 $\beta$ in human fibroblast-like synoviocytes and the effect of paeoniflorin. Int Immunopharmacol 12(4): 701-706, 2012. PMID: 22333895. DOI: 10.1016/j.intimp.2012.01.018 
37 Lin Z, Zhou P, von Gise A, Gu F, Ma Q, Chen J, Guo H, van Gorp PR, Wang DZ and Pu WT: Pi3kcb links Hippo-YAP and PI3K-AKT signaling pathways to promote cardiomyocyte proliferation and survival. Circ Res 116(1): 35-45, 2015. PMID: 25249570. DOI: 10.1161/CIRCRESAHA.115.304457

38 Lin Z, Tian XY, Huang XX, He LL and Xu F: microRNA-186 inhibition of PI3K-AKT pathway via SPP1 inhibits chondrocyte apoptosis in mice with osteoarthritis. J Cell Physiol 234(5): 6042-6053, 2019. PMID: 30500068. DOI: 10.1002/jcp.27225

39 Ediriweera MK, Tennekoon KH and Samarakoon SR: Role of the $\mathrm{PI} 3 \mathrm{~K} / \mathrm{AKT} / \mathrm{mTOR}$ signaling pathway in ovarian cancer: Biological and therapeutic significance. Semin Cancer Biol 59: 147-160, 2019. PMID: 31128298. DOI: 10.1016/j.semcancer.2019.05.012

40 Dey N, De P and Leyland-Jones B: PI3K-AKT-mTOR inhibitors in breast cancers: From tumor cell signaling to clinical trials. Pharmacol Ther 175: 91-106, 2017. PMID: 28216025. DOI: 10.1016/j.pharmthera.2017.02.037

41 Liu S, Cao C, Zhang Y, Liu G, Ren W, Ye Y and Sun T: PI3K/Akt inhibitor partly decreases TNF- $\alpha$-induced activation of fibroblast-like synoviocytes in osteoarthritis. J Orthop Surg Res 14(1): 425, 2019. PMID: 31829201. DOI: 10.1186/s13018019-1394-4

42 Kovacs JJ, Hara MR, Davenport CL, Kim J and Lefkowitz RJ: Arrestin development: emerging roles for beta-arrestins in developmental signaling pathways. Dev Cell 17(4): 443-458, 2009. PMID: 19853559. DOI: 10.1016/j.devcel.2009.09.011
43 Hara MR, Kovacs JJ, Whalen EJ, Rajagopal S, Strachan RT, Grant W, Towers AJ, Williams B, Lam CM, Xiao K, Shenoy SK, Gregory SG, Ahn S, Duckett DR and Lefkowitz RJ: A stress response pathway regulates DNA damage through $\beta 2$ adrenoreceptors and $\beta$-arrestin-1. Nature 477(7364): 349-353, 2011. PMID: 21857681. DOI: $10.1038 /$ nature 10368

44 Chen X, Zhang J, Xia L, Wang L, Li H, Liu H, Zhou J, Feng Z, Jin H, Yang J, Yang Y, Wu B, Zhang L, Chen G and Wang G: $\beta$ Arrestin-2 attenuates hepatic ischemia-reperfusion injury by activating PI3K/Akt signaling. Aging (Albany NY) 13(2): 22512263, 2020. PMID: 33323551. DOI: 10.18632/aging.202246

45 Sun X, Zhang Y, Wang J, Wei L, Li H, Hanley G, Zhao M, Li Y and Yin D: Beta-arrestin 2 modulates resveratrol-induced apoptosis and regulation of Akt/GSK3ß pathways. Biochim Biophys Acta 1800(9): 912-918, 2010. PMID: 20457218. DOI: 10.1016/j.bbagen.2010.04.015
Received March 10, 2021

Revised April 12, 2021

Accepted April 22, 2021 